\title{
Disseminated Mycobacterium abscessus subsp. massiliense infection in a Good's syndrome patient negative for human immunodeficiency virus and anti- interferon- $\gamma$ autoantibody: a case report
}

Waki Imoto ${ }^{1,2,3,4}$, Koichi Yamada ${ }^{1,2,4}$, Yuriko Hajika ${ }^{5}$, Kousuke Okamoto ${ }^{6}$, Yuka Myodo ${ }^{4}$, Makoto Niki ${ }^{4}$, Gaku Kuwabara 1,2,3,4, Kazushi Yamairi 1,2,4, Wataru Shibata ${ }^{1,2,4}$, Naoko Yoshii ${ }^{1,2,3,4}$, Kiyotaka Nakaie 1,4, Kazutaka Yoshizawa ${ }^{7}$, Hiroki Namikawa ${ }^{1,8}$, Tetsuya Watanabe ${ }^{3}$, Kazuhisa Asai ${ }^{3}$, Hiroshi Moro ${ }^{7}$, Yukihiro Kaneko $^{9}$, Tomoya Kawaguchi ${ }^{3}$, Yoshiaki Itoh ${ }^{6}$ and Hiroshi Kakeya ${ }^{1,24^{*}}$ (i)

\begin{abstract}
Background: Good's syndrome (GS) is characterized by immunodeficiency, and can lead to severe infection, which is the most significant complication. Although Mycobacterium rarely causes infection in patients with GS, disseminated nontuberculous mycobacterial (NTM) infection frequently occurs in GS patients that are also positive for the human immunodeficiency virus (HIV) or anti-interferon (IFN)- $\gamma$ autoantibodies. Here, we report a rare case of GS with NTM without HIV or IFN- $\gamma$ autoantibodies.
\end{abstract}

Case presentation: A 57-year-old Japanese male with GS and myasthenia gravis (treated with prednisolone and tacrolimus) was diagnosed with disseminated NTM infection caused by Mycobacterium abscessus subsp. massiliense. He presented with fever and back pain. Blood, lumbar tissue, urine, stool, and sputum cultures tested positive for $M$. abscessus. Bacteremia, spondylitis, intestinal lumber abscess, and lung infection were confirmed by bacteriological examination and diagnostic imaging; urinary and intestinal tract infections were suspected by bacteriological examination but not confirmed by imaging. Despite multidrug combination therapy, including azithromycin, imipenem/cilastatin, levofloxacin, minocycline, linezolid, and sitafloxacin, the patient ultimately died of the infection. The patient tested negative for HIV and anti-IFN- $\gamma$ autoantibodies.

Conclusions: Since myasthenia gravis symptoms interfere with therapy, patients with GS and their physicians should carefully consider the antibacterial treatment options against disseminated NTM.

Keywords: Anti-interferon-gamma autoantibody, Disseminated, Good's syndrome, Mycobacterium abscessus, Nontuberculous mycobacterial infection

\footnotetext{
* Correspondence: kakeya@med.osaka-cu.ac.jp

'Department of Infection Control Science, Osaka City University Graduate School of Medicine, 1-4-3, Asahi-machi, Abeno-ku, Osaka 545-8585, Japan

${ }^{2}$ Department of Infectious Disease Medicine, Osaka City University Hospital,

1-5-7 Asahi-machi, Abeno-ku, Osaka 545-8586, Japan

Full list of author information is available at the end of the article
}

(c) The Author(s). 2020 Open Access This article is licensed under a Creative Commons Attribution 4.0 International License, which permits use, sharing, adaptation, distribution and reproduction in any medium or format, as long as you give appropriate credit to the original author(s) and the source, provide a link to the Creative Commons licence, and indicate if changes were made. The images or other third party material in this article are included in the article's Creative Commons licence, unless indicated otherwise in a credit line to the material. If material is not included in the article's Creative Commons licence and your intended use is not permitted by statutory regulation or exceeds the permitted use, you will need to obtain permission directly from the copyright holder. To view a copy of this licence, visit http://creativecommons.org/licenses/by/4.0/. The Creative Commons Public Domain Dedication waiver (http://creativecommons.org/publicdomain/zero/1.0/) applies to the data made available in this article, unless otherwise stated in a credit line to the data. 


\section{Background}

Disseminated nontuberculous mycobacterial (NTM) infection is often associated with an infection by the human immunodeficiency virus (HIV) [1]. Anti-interferongamma (IFN- $\gamma$ ) autoantibodies are typically found in patients with disseminated NTM infection without HIV infection, especially in the Asian population [1, 2]. Good's syndrome (GS) is characterized by thymoma, hypogammaglobulinemia, and multiple infections [3]. Despite a few cases of Mycobacterium infection associated with thymoma, to the best of our knowledge, there has been no report of Mycobacterium associated with GS [4-6]. Herein, we describe a rare case of GS with disseminated NTM infection.

\section{Case presentation}

The patient was a 57-year-old Japanese male diagnosed with GS (thymoma and hypogammaglobulinemia), myasthenia gravis with anti-striational antibodies, and type 2 diabetes. Prednisolone (PSL) and tacrolimus (TAC) were used to treat the myasthenia gravis for more than 5 years, and his thymoma was removed at the age of 27 years. His father had been treated for lung Mycobacterium tuberculosis infection. The patient smoked for 27 years (since his twenties) and was a social drinker. He had worked in specimen processing at a specimen inspection company. His daily routine involved spending most of the day in bed and required assistance with his wheelchair and meals.

The patient presented with fever and back pain 1 month before his outpatient visit at the Department of Neurology. He was hospitalized during his regular visit, at which point blood and sputum samples were collected for culture, and he was administered tazobactam/piperacillin (TAZ/PIPC) and immunoglobulin by his primary care physician. The sputum smear was positive for acid-fast bacilli; chest computed tomography showed a suspected lung NTM infection and lumbar intestinal abscess, and magnetic resonance imaging revealed spondylitis (lumbar segments 1-2) during hospitalization (Fig. 1). Infective

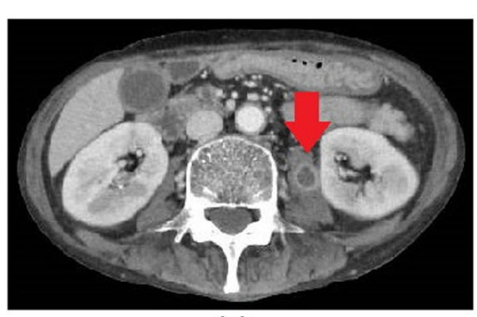

(a)

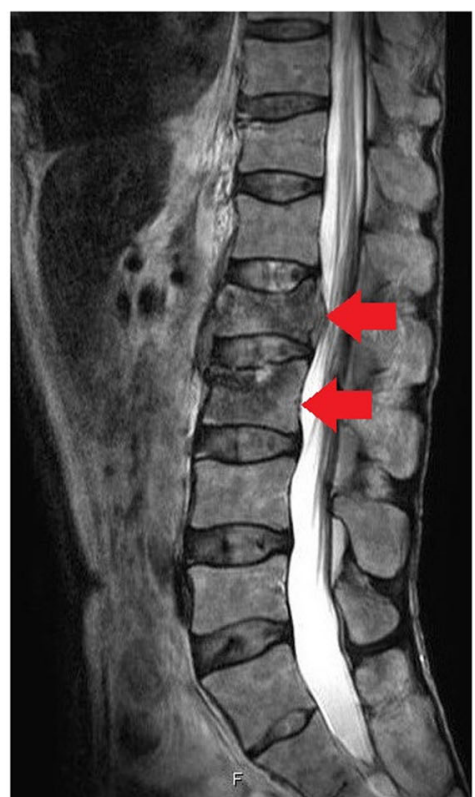

(c)

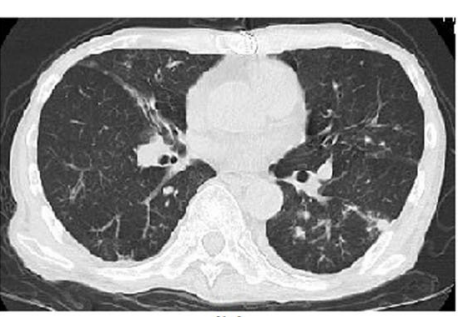

(b)

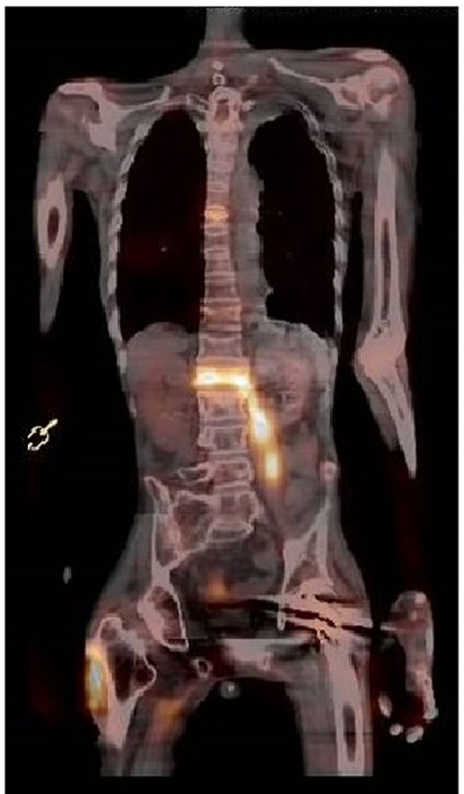

(d)

Fig. 1 a Contrast-enhanced computed tomography scan of the abdomen showing the left iliopsoas abscess (red arrow). b Chest computed tomography showing the scattered nodules. c Contrast-enhanced magnetic resonance imaging of the spine (T2) showing pyogenic spondylitis at lumbar segments 1 and 2 (red arrows). d Gallium scintigraphy showing the accumulation of salt in the lumbar spine and iliopsoas muscle 
endocarditis was not detected by transthoracic echocardiography. Mycobacterial infection was suspected, and blood culture was performed on day 5 of hospitalization. His general condition and vitals were stable, and the TAZ/PIPC treatment was continued while awaiting empirical therapy for Mycobacteria. Two days later, blood and sputum cultures revealed the presence of Mycobacterium abscessus, resulting in a diagnosis of disseminated NTM infection.

The patient was transferred to the Department of Infectious Disease and was screened for immunodeficiency. He tested negative for HIV-specific antibodies, and the blood samples sent to Nigata University tested negative for antiIFN- $\gamma$ autoantibodies. The conclusive diagnosis of $M$. abscessus subsp. massiliense infection was the result of a combination of $16 \mathrm{~S}$ ribosomal RNA sequencing and nucleic acid chromatography of the RNA polymerase $B$ and $h s p 65$ genes. He underwent a lumbar biopsy on day 8 after hospitalization and was prescribed empiric therapy with imipenem (IPM)/cilastatin, levofloxacin, and azithromycin.
The antibiotics were continued since $M$. abscessus was also detected in the biopsy tissue, urine, and stool cultures.

The patient developed a gastrointestinal (rectum and descending colon) perforation on day 15 of hospitalization and underwent surgery (high anterior resection, left hemicolectomy, colostomy, and abdominal drainage). Despite the continued use of antibiotics, his spondylitis worsened. Minocycline (MINO) and linezolid (LZD) were included in the antibiotic regimen on day 17 and 24, respectively. Finally, after levofloxacin was substituted with sitafloxacin (STFX), the antibiotic combination of IPM/ CS, STFX, azithromycin, MINO, and LZD was continued (Fig. 2). Although the sensitivity of $M$. abscessus subsp. massiliense was detected by microdilution [7, 8], the strain showed a different susceptibility towards each antibiotic (Table 1), and the patient's general condition worsened. The patient and his family were informed of the treatment options, and after obtaining consent, he was moved to palliative care. He passed away on day 49 in the hospital.

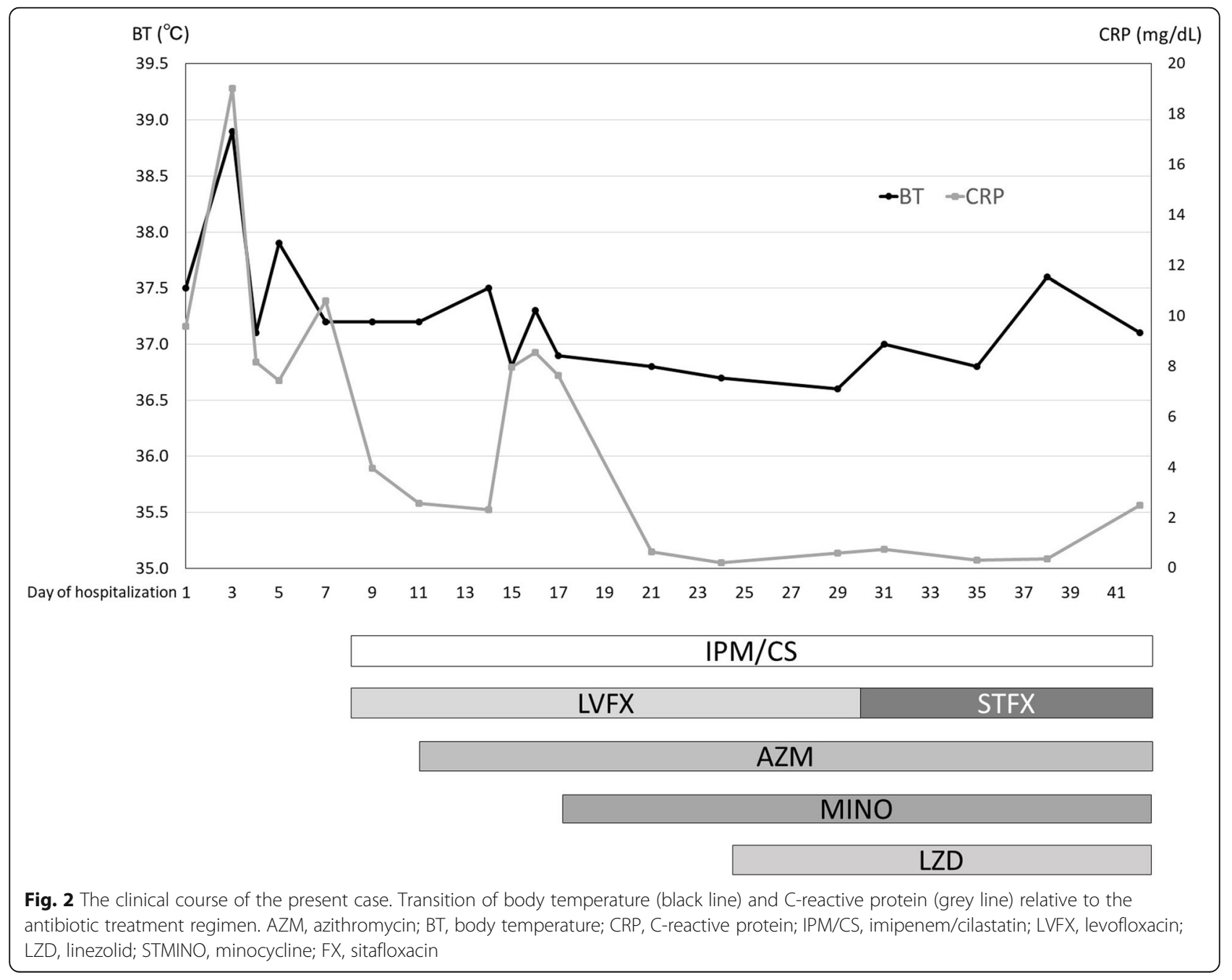


Table 1 Antibiotic susceptibility of the identified Mycobacterium abscessus subsp. massiliense clinical isolate

\begin{tabular}{lllll}
\hline Antibiotic & \multicolumn{2}{l}{ Minimum Inhibitory Concentration Breakpoints (mg/L) [7] } & $\begin{array}{l}\text { Minimum Inhibitory } \\
\text { Concentration (mg/L) }\end{array}$ \\
\cline { 2 - 5 } & Susceptibility & Intermediate & Resistance & 8 \\
\hline Clarithromycin & $\leqq 2$ & 4 & $\geqq 8$ & 16 \\
Imipenem & $\leqq 4$ & $8-16$ & $\geqq 32$ & $>64$ \\
Meropenem & $\leqq 4$ & $8-16$ & $\geqq 8$ & $>8$ \\
Minocycline & $\leqq 1$ & $2-4$ & $\geqq 64$ & 16 \\
Amikacin & $\leqq 16$ & 32 & $\geqq 4$ & $>8$ \\
Moxifloxacin & $\leqq 1$ & 2 & $\geqq 32$ & 32 \\
Linezolid & $\leqq 8$ & 16 & $\geqq 4 / 76$ & $>8 / 152$ \\
Trimethoprim-sulfamethoxazole & $\leqq 2 / 38$ & - & & \\
\hline
\end{tabular}

\section{Discussion and conclusions}

Infections are the most frequent and important complications associated with GS [9]. Previous reports have shown that the upper and lower respiratory tracts are the most common sites of infection in patients with GS [10], and bacterial infections are the most significant [9, 10]. However, infections by Mycobacterium are rare in GS patients, and only a few cases of Mycobacterium infection associated with thymoma (without GS) have been reported [4-6, 11]. We surveyed the literature, and the relevant reports are listed in Table 2 . To the best of our knowledge, this is the first report of a Mycobacterium infection associated with GS.

A common variable immune deficiency like GS is characterized by hypogammaglobulinemia, which increases the risk of infection by bacteria and certain viruses [12]. In particular, infection by cytomegaloviruses and Pneumocystis jirovecii is rampant in GS patients since their immune cells are depleted; this is a common characteristic of GS that distinguishes it from common variable immune deficiency $[13,14]$. Despite the depletion of immune cells, as the major host defense mechanism, mycobacterial infections are nevertheless rare in GS patients [15]; however, the reason remains to be elucidated [11]. The severe disseminated NTM infection in our GS patient might have been induced by the several treatments he was administered. The patient had myasthenia gravis, which was treated with PSL and TAC. These immunosuppressive agents are known to induce cell-mediated immunodeficiency, which might have triggered the mycobacterial infection [16].

Treatment was difficult in the present case because of the immunodeficiency and side effects of the drugs. Liu and $\mathrm{Hu}$ [17] reported that aminoglycosides might worsen myasthenia gravis by competitively inhibiting the release of acetylcholine from the presynaptic membranes, thereby impairing depolarization of the postsynaptic membrane and reducing irritability of the myocyte membrane around the end-plate membrane that subsequently leads to blockade of the neuromuscular junction. Thus, even though aminoglycosides are very potent antimicrobial agents, they could not be used to treat our patient [18]. Moreover, in the past, M. abscessus was reported to show the lowest macrolide resistance rate [19]. However, in this case, the strain showed a high MIC $(\mathrm{MIC}=8)$ value for clarithromycin, which remarkably challenged treatment. There is no clear association between the intestine perforation and Mycobacterium

Table 2 Past reports of thymoma (with or without Good's syndrome) with Mycobacterium infection

\begin{tabular}{llll}
\hline & This case & Murakami O, et al. [4] & Satoh H, et al. [5] \\
\hline GS or thymoma & GS & thymoma & $\begin{array}{l}\text { thymoma (GS suspected, } \\
\text { not detected) } \\
\text { age }\end{array}$ \\
sex & 57 y & 33 y & 69 y \\
Infectious microorganism & Male & Male & Male \\
treatment for infection & IPM, AZM, MINO, LZD, STFX & Mycobacterium tuberculosis suspected & Mycobacterium intracellulare \\
complications & DM, anti-rhabdomyolysis, antibody- & INH, RFP, SM & observation \\
& positive myasthenia gravis & & not particular \\
immunosuppressants & PSL, TAC & none & none \\
year & 2019 & 1998 & 2012 \\
\hline
\end{tabular}

AZM azithromycin, DM diabetes mellitus, GS Good's syndrome, INH Isoniazid, IPM imipenem, LZD linezolid, MINO minocycline, PSL prednisolone, RFP Rifampicin, SM Streptomycin

TAC: tacrolimus 
infection of the present case. The resected specimen did not show any abnormalities; thus, Mycobacterium or other infections could not be diagnosed. Since $M$. abscessus was detected in the stool culture, it is still possible that $M$. abscessus caused the intestinal perforation.

In conclusion, this is the first case of disseminated NTM infection in a GS patient without infection by HIV or the production of anti-IFN- $\gamma$ autoantibodies. Although NTM infection can occur in GS patients, the treatment for $M$. abscessus infection is difficult in such cases and should be carefully considered, since aminoglycosides cannot be used in GS.

\section{Abbreviations}

GS: Good's syndrome; HIV: Human immunodeficiency virus; IFN-ү: Interferongamma; INH: Isoniazid; IPM: Imipenem; LZD: Linezolid; MINO: Minocycline; NTM: Nontuberculous mycobacterial; PSL: Prednisolone; RFP: Rifampicin SM: Streptomycin; TAC: Tacrolimus; TAZ/PIPC: Tazobactam/piperacillin

\section{Acknowledgments}

We would like to thank Editage (www.editage.com) for their assistance with English language editing.

\section{Authors' contributions}

WI was the primary doctor of the patient and edited the case presentation. KY provided feedback regarding treatment as a senior fellow of the infectious disease medicine department. YH participated in the treatment as a trainee doctor. $\mathrm{KO}$ provided expert opinion and was involved in the treatment of the neurological disease. KY, GK, KY2, WS, and NY participated in the treatment as members of the department of infectious disease medicine. YM, MN and KN identified the Mycobacterium species. KY3 and $\mathrm{HM}$ tested for anti-interferon- $\gamma$ autoantibodies. HN, TW, KA, TK, YI, YK, and HK provided valuable feedback for the report. All the authors read and approved the final manuscript.

\section{Funding}

This research did not receive any specific grant from funding agencies in the public, commercial, or not-for-profit sectors.

\section{Availability of data and materials}

The data that support the findings of this study are available from the corresponding author upon reasonable request.

\section{Ethics approval and consent to participate}

Not applicable.

\section{Consent for publication}

Written informed consent was obtained from the patient's family for publication of this case report and the accompanying images. A copy of the written consent is available for review upon request

\section{Competing interests}

The authors declare that they have no competing interests.

\section{Author details}

'Department of Infection Control Science, Osaka City University Graduate School of Medicine, 1-4-3, Asahi-machi, Abeno-ku, Osaka 545-8585, Japan. ${ }^{2}$ Department of Infectious Disease Medicine, Osaka City University Hospital, 1-5-7 Asahi-machi, Abeno-ku, Osaka 545-8586, Japan. ${ }^{3}$ Department of Respiratory Medicine, Osaka City University Graduate School of Medicine, 1-4-3 Asahi-machi, Abeno-ku, Osaka 545-8585, Japan. ${ }^{4}$ Department of Infection Control and Prevention, Osaka City University Hospital, 1-5-7 Asahi-machi, Abeno-ku, Osaka 545-8586, Japan. ${ }^{5}$ Department of Metabolism, Endocrinology, Osaka City University Graduate School of Medicine, 1-4-3 Asahi-machi, Abeno-ku, Osaka 545-8585, Japan. 'Department of Neurology, Osaka City University Graduate School of Medicine, 1-4-3 Asahi-machi, Abeno-ku, Osaka 545-8585, Japan. ${ }^{7}$ Department of Respiratory Medicine and Infectious Diseases, Niigata University Graduate School of Medical and Dental
Sciences, 1-757 Asahimachi-dori, Chuo-ku, Niigata 951-8510, Japan. ${ }^{8}$ Department of Medical Education and General Practice, Osaka City University Graduate School of Medicine, 1-4-3 Asahi-machi, Abeno-ku, Osaka 545-8585, Japan. ${ }^{9}$ Department of Bacteriology, Osaka City University Graduate School of Medicine, 1-4-3 Asahi-machi, Abeno-ku, Osaka 545-8585, Japan.

Received: 18 January 2020 Accepted: 4 June 2020

Published online: 20 June 2020

\section{References}

1. Chetchotisakd P, Kiertiburanakul S, Mootsikapun P, Assanasen S, Chaiwarith $\mathrm{R}$, Anunnatsiri S. Disseminated nontuberculous mycobacterial infection in patients who are not infected with HIV in Thailand. Clin Infect Dis. 2007;45: 421-7.

2. Kampitak T, Suwanpimolkul G, Browne S, Suankratay C. Anti-interferon-y autoantibody and opportunistic infections: case series and review of the literature. Infection. 2011;39:65-71.

3. Dong JP, Gao W, Teng GG, Tian Y, Wang HH. Characteristics of Good's syndrome in China: a systematic review. Chin Med J. 2017;130:1604-9.

4. Murakami O, Satoh H, Ohtsuka M, Funayama Y, Hasegawa S, Ishikawa H, et al. The coexistence of pulmonary tuberculosis and thymoma a case report. Kekkaku. 1998;73:525-9 [in Japanese].

5. Satoh H, Kagohashi K, Ohara G, Miyazaki Z, Kawaguchi M, Kurishima K, et al. A case of thymoma and Mycobacterium intracellular infection. Kekkaku. 2012:87:701-5 [in Japanese].

6. Tachdjian R, Keller JJ, Pfeffer M. A bad case of Good's syndrome. Infect Dis Ther. 2014;3:333-7.

7. CLSI, editor. Susceptibility testing of mycobacteria, Nocardia spp., and other aerobic actinomycetes, $3^{\text {rd }}$ ed. CLSI standard M24. Wayne: Clinical and Laboratory Standard Institute; 2018.

8. CLSI, editor. Performance standards for susceptibility testing of mycobacteria, Nocardia spp., and other aerobic actinomycetes, $1^{\text {st }}$ ed. CLSI supplement M62. Wayne: Clinical and Laboratory Standard Institute; 2018.

9. Sun X, Shi J, Wang M, Xu K, Xiao Y. Good's syndrome patients hospitalized for infections: a single-center retrospective study. Medicine. 2015;94:e2090

10. Tarr PE, Sneller MC, Mechanic LJ, Economides A, Eger CM, Strober W, et al. Infections in patients with immunodeficiency with thymoma (Good syndrome). Report of 5 cases and review of the literature. Medicine. 2001;80: 123-33.

11. Kikuchi R, Mino N, Okamoto T, Matsukara T, Hirai T. A case of Good's syndrome: a rare acquired immunodeficiency associated with thymoma. Ann Thorac Cardiovasc Surg. 2011;17:74-6.

12. Bonilla FA, Geha RS. Common variable immunodeficiency. Pediatr Res. 2009; 65:13-9.

13. Cunningham-Rundles C, Bodian C. Common variable immunodeficiency: clinical and immunological features of 248 patients. Clin Immunol. 1999;92: 34-48.

14. Gathmann B, Mahlaoui N, CEREDIH, Gérard L, Oksenhendler E, Warnatz K, et al. Clinical picture and treatment of 2212 patients with common variable immunodeficiency. J Allergy Clin Immunol. 2014;134:116-26.

15. Dannenberg AM Jr. Delayed-type hypersensitivity and cell-mediated immunity in the pathogenesis of tuberculosis. Immunol Today. 1991;12:22833.

16. Azar AE, Ballas ZK. Evaluation of the adult with suspected immunodeficiency. Am J Med. 2007;120:764-8.

17. Liu C, Hu F. Investigation on the mechanism of exacerbation of myasthenia gravis by aminoglycoside antibiotics in mouse model. J Huazhong Univ Sci Technolog Med Sci. 2005;25:294-6.

18. Griffith DE, Aksamit T, Brown-Elliott BA, Catanzaro A, Daley C, Gordin F, et al. An official ATS/IDSA statement: diagnosis, treatment, and prevention of nontuberculous mycobacterial diseases. Am J Respir Crit Care Med. 2007; 175:367-416.

19. Shen $Y$, Wang $X$, Jin J, Wu J, Zhang $X$, Chen J, et al. In vitro susceptibility of Mycobacterium abscessus and Mycobacterium fortuitum isolates to 30 antibiotics. Biomed Res Int. 2018;2018:4902941.

\section{Publisher's Note}

Springer Nature remains neutral with regard to jurisdictional claims in published maps and institutional affiliations. 